\title{
APPLICATION OF SOCIAL COGNITIVE THEORY ON THE DETERMINANTS OF EXCLUSIVE BREASTFEEDING PRACTICE IN MADIUN, EAST JAVA
}

\author{
Rezyana Budi Syahputri'), Pawito²), Bhisma Murti1) \\ ${ }^{1)}$ Masters Program in Public Health, Universitas Sebelas Maret \\ 2)Faculty of Social and Political Sciences, Universitas Sebelas Maret
}

\begin{abstract}
Background: Exclusive breastfeeding can reduce child mortality. The achievement of exclusive breastfeeding has not reached the expected number due to various factors. This study aims to examine the determinants of exclusive breastfeeding practice in Madiun, East Java.

Subjects and Method: A cross-sectional study was carried out in Madiun, from February to May 2020. A sample of 200 lactating mothers was selected randomly. The dependent was exclusive breastfeeding. The independent variables were health promotion program, observational learning, role model, vicarious learning, imitation, positive attitude, outcome expectation, self-regulation, self-efficacy, and reinforcing. The data were collected by questionnaire and analyzed by a multiple logistic regression run on Stata 13 .

Results: Exclusive breastfeeding increased with following health promotion in exclusive breastfeeding program $(\mathrm{OR}=2.76 ; 95 \% \mathrm{CI}=1.05$ to $7.25 ; \mathrm{p}=0.039)$, high observational learning $(\mathrm{OR}=3.33 ; 95 \% \mathrm{CI}=1.27$ to $8.71 ; \mathrm{p}=0.014)$, strong role model $(\mathrm{OR}=3.43 ; 95 \%$ $\mathrm{CI}=1.10$ to $10.67 ; \mathrm{p}=0.033)$, high vicarious learning $(\mathrm{OR}=3.83 ; 95 \% \mathrm{CI}=1.31$ to $11.12 ; \mathrm{p}=$ 0.014), strong imitation $(\mathrm{OR}=2.98 ; 95 \% \mathrm{CI}=1.19$ to $7.48 ; \mathrm{p}=0.020)$, positive attitude $(\mathrm{OR}=3.14 ; 95 \% \mathrm{CI}=1.03$ to $9.60 ; \mathrm{p}=0.044)$, positive outcome expectation $(\mathrm{OR}=3.56 ; 95 \%$ $\mathrm{CI}=1.30$ to $9.79 ; \mathrm{p}=0.014)$, strong self-regulation $(\mathrm{OR}=2.59 ; 95 \% \mathrm{CI}=1.10$ to $6.07 ; \mathrm{p}=$ o.028), strong self-efficacy $(\mathrm{OR}=4.91 ; 95 \% \mathrm{CI}=1.84$ to $13.11 ; \mathrm{p}=0.001)$, and reinforcing $(\mathrm{OR}=3.42 ; 95 \% \mathrm{CI}=1.17$ to $10.01 ; \mathrm{p}=0.024)$.

Conclusion: Exclusive breastfeeding increases with following health promotion in exclusive breastfeeding program, high observational learning, strong role model, high vicarious learning, strong imitation, positive attitude, positive outcome expectation, strong self-regulation, strong self-efficacy, and reinforcing.
\end{abstract}

Keywords: exclusive breastfeeding, health promotion, social cognitive theory

\section{Correspondence:}

Rezyana Budi Syahputri. Masters Program in Public Health, Universitas Sebelas Maret, Jl. Ir. Sutami 36A, Surakarta 57126, Central Java. Email: rezyanabs@gmail.com. Mobile: $+6282325750134$ 